\title{
Neutron matter on the lattice with pionless effective field theory
}

\author{
Dean Lee \\ Department of Physics, North Carolina State University, Raleigh, NC 27695 \\ Thomas Schäfer \\ Department of Physics, North Carolina State University, Raleigh, NC 27695 \\ RIKEN-BNL Research Center, Brookhaven National Laboratory, Upton, NY 11973
}

\begin{abstract}
We study neutron matter by combining pionless effective field theory with non-perturbative lattice methods. The neutron contact interaction is determined by zero temperature scattering data. We simulate neutron matter on the lattice at temperatures 4 and $8 \mathrm{MeV}$ and densities below one-fifth normal nuclear matter density. Our results at different lattice spacings agree with one another and match bubble chain calculations at low densities. The equation of state of pure neutron matter obtained from our simulations agrees quantitatively with variational calculations based on realistic potentials.
\end{abstract}




\section{INTRODUCTION}

The equation of state of dilute neutron matter is of central importance to the structure and evolution of neutron stars 1, 2]. In addition to that, the neutron matter problem has many interesting physical aspects. Neutron matter has positive pressure at all densities and becomes a superfluid at sufficiently small temperature. If the density is small, pairing is expected to take place in an $S$-wave, but at higher density $P$-wave pairing might be dominant [3]. The neutron matter problem contains a number of very different scales. The neutron scattering length is very large, $a_{n n} \simeq-18 \mathrm{fm}$, which implies that the dimensionless parameter $k_{F}\left|a_{n n}\right| \gg 1$ for densities $\rho>10^{-4} \rho_{N}$. Here, $k_{F}=\left(3 \pi^{2} \rho\right)^{1 / 3}$ is the Fermi momentum and $\rho_{N} \simeq 0.17 \mathrm{fm}^{-3}$ is the saturation density of nuclear matter. The effective range, on the other hand, is of natural size, $r_{n n} \simeq 2.8 \mathrm{fm}$. As a consequence, the parameter $k_{F}\left|r_{n n}\right|$ is neither large nor small for densities $\rho \sim \rho_{N}$.

If the density is very small, $\rho<0.1 \rho_{N}$, then $k_{F}\left|r_{n n}\right|$ is a small parameter and neutron matter is close to the limit in which $k_{F}\left|a_{n n}\right| \rightarrow \infty$ and $k_{F}\left|r_{n n}\right| \rightarrow 0$. In this limit dimensional analysis implies that the energy per particle and the gap have to be proportional to the Fermi energy

$$
\frac{E}{A}=\xi \frac{3}{5} \frac{k_{F}^{2}}{2 m}, \quad \Delta=\zeta \frac{k_{F}^{2}}{2 m} .
$$

The determination of the two dimensionless parameters $\xi$ and $\zeta$ is a fascinating nonperturbative problem that has received a lot of attention recently. This interest was fueled by experimental advances in creating cold, dilute gases of fermionic atoms tuned to be near a Feshbach resonance [4, [5, 6, 7, [8].

The traditional approach to the neutron matter problem is based on the assumption that nucleons can be treated as non-relativistic point-particles interacting mainly via two-body potentials. The two-body potentials are fitted to experimental data on nucleon-nucleon scattering. The many-body problem is addressed by solving the many-body Schrödinger equation using variational methods or Green function Monte Carlo methods guided by variational wave functions [9, 10].

Even though this method has been very successful it is desirable to seek an alternative approach that is more directly related to QCD, systematically improvable, and that lends itself to numerical studies which do not rely on variational wave functions. Such an approach is provided by effective field theory. The use of effective field theory (EFT) methods in 
nuclear physics was pioneered by Weinberg [11]. Over the last few years EFT methods have been applied successfully to the study of two and three-body systems at low energy 12, 13, 14]. Nuclear and neutron matter was studied using a perturbative expansion in powers of the Fermi momentum [15] and using lattice simulations [16, 17].

In this paper we shall study dilute neutron matter using a nuclear effective field theory on the lattice. Since we are interested in densities below nuclear matter saturation density we shall assume that the relevant momenta are smaller than the pion mass and that we can use an effective field theory that contains only neutrons. In this work we shall limit ourselves to the lowest order effective Lagrangian which contains a single four-fermion contact interaction with no derivatives that is adjusted to the neutron-neutron scattering length. This effective theory is sufficient in order to investigate universal properties in the limit $k_{F}\left|a_{n n}\right| \rightarrow \infty$, $k_{F}\left|r_{n n}\right| \rightarrow 0$. An important advantage of the model is the fact that in the case of an attractive interaction there is no sign problem at finite density [18]. As a consequence, the theory can be simulated efficiently using standard hybrid Monte Carlo algorithms [19].

The paper is organized as follows. In Sects. II-IV we introduce the lattice theory. In Sect. $\mathrm{V}$ we discuss how to determine the coefficient of the four fermion interaction by matching to the two-body scattering length. In Sect. VI we study a low density approximation to the partition function based on summing particle-particle chains. In Sect. VII we describe our hybrid Monte Carlo method. Numerical results for the neutron density, the energy per particle and the equation of state are given in Sects. VIII-XII.

\section{NOTATION}

Before describing the physics we first define some notation we use throughout our discussion. We let $\vec{n}$ represent integer-valued lattice vectors on our $3+1$ dimensional space-time lattice. We use a subscripted " $s$ " such as in $\vec{n}_{s}$ to represent purely spatial lattice vectors. We use subscripted indices such as $i, j$ for the two spin components of the neutron, $\uparrow$ and $\downarrow$. We let $\hat{0}$ be the unit lattice vector in the time direction and let $\hat{l}_{s}=\hat{1}, \hat{2}, \hat{3}$ be the corresponding unit lattice vectors in the spatial directions. A summation symbol such as

$$
\sum_{l_{s}}
$$

implies a summation over values $l_{s}=1,2,3$. 
We take the neutron mass to be $939 \mathrm{MeV}$, and normal nuclear matter density to be 0.17 $\mathrm{fm}^{-3}$. We let $a$ be the lattice spacing in the spatial direction and $L$ be the number of lattice sites in each spatial direction. $a_{t}$ is the lattice spacing in the temporal direction and $L_{t}$ is the number of lattice sites in the temporal direction. We let $\alpha_{t}$ be the ratio between lattice spacings,

$$
\alpha_{t}=\frac{a_{t}}{a}
$$

Throughout we use dimensionless parameters and operators, which correspond with physical values multiplied by the appropriate power of $a$. In the end, however, we report final results in physical units such as $\mathrm{MeV}$ or $\mathrm{fm}^{-3}$. In cases where there may be confusion, we use the subscript phys to identify quantities in physical units.

We use $a, a^{\dagger}$ to represent annihilation and creation operators for the neutron, whereas $c, c^{*}$ indicate the corresponding Grassmann variables in the path integral representation. We let $m_{N}$ be the mass of the neutron and $\mu$ be the neutron chemical potential. For the neutron fields we apply periodic boundary conditions in the spatial directions and antiperiodic boundary conditions in the temporal direction. For each neutron momentum we use the notation

$$
\vec{k}_{*}=\left(\frac{2 \pi}{L_{t}} k_{0}, \frac{2 \pi}{L} k_{1}, \frac{2 \pi}{L} k_{2}, \frac{2 \pi}{L} k_{3}\right)
$$

where $k_{1}, k_{2}$, and $k_{3}$ are integers and $k_{0}$ is an odd half-integer. In physical units the momentum is

$$
\vec{k}_{\text {phys }}=\left(k_{* 0} a_{t}^{-1}, k_{* 1} a^{-1}, k_{* 2} a^{-1}, k_{* 3} a^{-1}\right) .
$$

Unless otherwise indicated, our the momentum labels will follow this convention. For convenience we also define

$$
h=\frac{\alpha_{t}}{2 m_{N}}
$$

and

$$
\omega_{k}=6 h-2 h \sum_{l_{s}} \cos \left(k_{* l_{s}}\right) .
$$

We let $D^{\text {free }}(\vec{k}) \delta_{i j}$ be the free neutron propagator. For notational convenience the spinconserving $\delta_{i j}$ in the neutron propagator will be implicit. The self-energy, $\Sigma(\vec{k})$, is defined by

$$
D^{\text {full }}(\vec{k})=\frac{D^{\text {free }}(\vec{k})}{1-\Sigma(\vec{k}) D^{\text {free }}(\vec{k})},
$$


where $D^{f u l l}(\vec{k})$ is the fully-interacting propagator.

In our plots we use the abbreviation "fc" for free continuum results, "f" for free lattice results, "b" for bubble chain calculations, and "s" for lattice simulations results. In addition to these abbreviations, we will use the shorthand labels shown in Table 1 for various combinations of spatial and temporal lattice spacings presented in our analysis.

Table 1: Shorthand labels for various lattice spacings used

\begin{tabular}{|l|l|l|}
\hline$a^{-1}(\mathrm{MeV})$ & $a_{t}^{-1}(\mathrm{MeV})$ & Label \\
\hline 50 & 24 & 0 \\
\hline 60 & 32 & 1 \\
\hline 60 & 48 & 2 \\
\hline 70 & 64 & 3 \\
\hline 80 & 72 & 4 \\
\hline
\end{tabular}

\section{FREE NUCLEON}

On the lattice the free neutron Hamiltonian can be written as

$$
\begin{aligned}
H_{\bar{N} N} & =\sum_{\vec{n}_{s}, i}\left[\left(m_{N}-\mu+\frac{3}{m_{N}}\right) a_{i}^{\dagger}\left(\vec{n}_{s}\right) a_{i}\left(\vec{n}_{s}\right)\right] \\
& -\frac{1}{2 m_{N}} \sum_{\vec{n}_{s}, l_{s}, i}\left[a_{i}^{\dagger}\left(\vec{n}_{s}\right) a_{i}\left(\vec{n}_{s}+\hat{l}_{s}\right)+a_{i}^{\dagger}\left(\vec{n}_{s}\right) a_{i}\left(\vec{n}_{s}-\hat{l}_{s}\right)\right] .
\end{aligned}
$$

We can approximate the partition function as a Euclidean lattice path integral,

$$
Z_{G}^{f r e e}=\operatorname{Tr} \exp \left[-\beta H_{\bar{N} N}\right] \simeq z_{0}^{\text {free }} \int D c D c^{*} \exp \left[-S^{\text {free }}\right]
$$

where $z_{0}^{\text {free }}$ is a constant and

$$
\begin{aligned}
S^{\text {free }} & =\sum_{\vec{n}, i}\left[c_{i}^{*}(\vec{n}) c_{i}(\vec{n}+\hat{0})-e^{-\left(m_{N}-\mu\right) \alpha_{t}}(1-6 h) c_{i}^{*}(\vec{n}) c_{i}(\vec{n})\right] \\
& -h e^{-\left(m_{N}-\mu\right) \alpha_{t}} \sum_{\vec{n}, l_{s}, i}\left[c_{i}^{*}(\vec{n}) c_{i}\left(\vec{n}+\hat{l}_{s}\right)+c_{i}^{*}(\vec{n}) c_{i}\left(\vec{n}-\hat{l}_{s}\right)\right]
\end{aligned}
$$

We have taken a slightly different form than that used in [17]. Instead of the $e^{-6 h}$ that appears in [17], we use the more standard $1-6 h$ as the coefficient multiplying $c_{i}^{*}(\vec{n}) c_{i}(\vec{n})$.

It is conventional to define a new normalization for $c_{i}$,

$$
c_{i}^{\prime}=c_{i} e^{-\left(m_{N}-\mu\right) \alpha_{t}}
$$


Then

$$
Z_{G}^{\text {free }} \simeq z_{0}^{\text {free }} e^{-2\left(m_{N}-\mu\right) \beta L^{3}} \int D c^{\prime} D c^{*} \exp \left[-S^{\text {free }}\right]
$$

where

$$
\begin{aligned}
S^{\text {free }} & =\sum_{\vec{n}, i}\left[e^{\left(m_{N}-\mu\right) \alpha_{t}} c_{i}^{*}(\vec{n}) c_{i}^{\prime}(\vec{n}+\hat{0})-(1-6 h) c_{i}^{*}(\vec{n}) c_{i}^{\prime}(\vec{n})\right] \\
& -h \sum_{\vec{n}, l_{s}, i}\left[c_{i}^{*}(\vec{n}) c_{i}^{\prime}\left(\vec{n}+\hat{l}_{s}\right)+c_{i}^{*}(\vec{n}) c_{i}^{\prime}\left(\vec{n}-\hat{l}_{s}\right)\right] .
\end{aligned}
$$

In momentum space we have

$$
S^{f r e e}=\sum_{\vec{k}, i} \tilde{c}_{i}^{*}(-\vec{k}) \tilde{c}_{i}^{\prime}(\vec{k})\left[e^{-i k_{* 0}+\left(m_{N}-\mu\right) \alpha_{t}}-(1-6 h)-2 h \sum_{l_{s}} \cos \left(k_{* l_{s}}\right)\right] .
$$

The free neutron correlation function on the lattice is

$$
\frac{\int D c^{\prime} D c^{*} c_{i}^{\prime}(\vec{n}) c_{i}^{*}(0) \exp \left[-S^{\text {free }}\right]}{\int D c^{\prime} D c^{*} \exp \left[-S^{\text {free }}\right]}=\frac{1}{L_{t} L^{3}} \sum_{\vec{k}} e^{-i \vec{k}_{*} \cdot \vec{n}} D^{\text {free }}(\vec{k})
$$

(no sum over $i$ ) where the free neutron propagator is

$$
\begin{aligned}
D^{\text {free }}(\vec{k}) & =\frac{1}{e^{-i k_{* 0}+\left(m_{N}-\mu\right) \alpha_{t}}-(1-6 h)-2 h \sum_{l_{s}} \cos \left(k_{* l_{s}}\right)} \\
& =\frac{1}{e^{-i k_{* 0}+\left(m_{N}-\mu\right) \alpha_{t}}-1+\omega_{k}}
\end{aligned}
$$

\section{NEUTRON CONTACT TERM}

There are two contact interactions at lowest order in the effective theory of nucleons without pions. But since we are considering pure neutron matter, this reduces to one contact interaction of the form

$$
H_{\bar{N} N \bar{N} N}=C \sum_{\vec{n}_{s}} a_{\uparrow}^{\dagger}\left(\vec{n}_{s}\right) a_{\uparrow}\left(\vec{n}_{s}\right) a_{\downarrow}^{\dagger}\left(\vec{n}_{s}\right) a_{\downarrow}\left(\vec{n}_{s}\right)
$$

Since

$$
\exp \left[-\frac{C \alpha_{t}}{2}\left(a_{\uparrow}^{\dagger} a_{\uparrow}+a_{\downarrow}^{\dagger} a_{\downarrow}\right)^{2}\right]=\sqrt{\frac{1}{2 \pi}} \int_{-\infty}^{\infty} d s \exp \left[-\frac{1}{2} s^{2}+s \sqrt{-C \alpha}\left(a_{\uparrow}^{\dagger} a_{\uparrow}+a_{\downarrow}^{\dagger} a_{\downarrow}\right)\right],
$$

we can write

$$
\exp \left[-C \alpha_{t} a_{\uparrow}^{\dagger} a_{\uparrow} a_{\downarrow}^{\dagger} a_{\downarrow}\right]=\sqrt{\frac{1}{2 \pi}} \int_{-\infty}^{\infty} d s \exp \left[-\frac{1}{2} s^{2}+\left(s \sqrt{-C \alpha}+\frac{C \alpha_{t}}{2}\right)\left(a_{\uparrow}^{\dagger} a_{\uparrow}+a_{\downarrow}^{\dagger} a_{\downarrow}\right)\right] .
$$


With this interaction the partition function can be approximated by

$$
Z_{G}=\operatorname{Tr} \exp \left[-\beta\left(H_{\bar{N} N}+H_{\bar{N} N \bar{N} N}\right)\right] \simeq z_{0} \int D s D c D c^{*} \exp [-S]
$$

where $z_{0}$ is a constant and

$$
\begin{aligned}
S & =\sum_{\vec{n}, i}\left[e^{\left(m_{N}-\mu\right) \alpha_{t}} c_{i}^{*}(\vec{n}) c_{i}^{\prime}(\vec{n}+\hat{0})-e^{\sqrt{-C \alpha_{t}} s(\vec{n})+\frac{C \alpha_{t}}{2}}(1-6 h) c_{i}^{*}(\vec{n}) c_{i}^{\prime}(\vec{n})\right] \\
& -h \sum_{\vec{n}, l_{s}, i}\left[c_{i}^{*}(\vec{n}) c_{i}^{\prime}\left(\vec{n}+\hat{l}_{s}\right)+c_{i}^{*}(\vec{n}) c_{i}^{\prime}\left(\vec{n}-\hat{l}_{s}\right)\right]+\frac{1}{2} \sum_{\vec{n}} s^{2}(\vec{n}) .
\end{aligned}
$$

This lattice action is quite simple, and in the future it may be worth considering improved actions in order to reduce discretization errors. Nevertheless our lattice action maintains some important properties. One property is that the chemical potential, $\mu$, is coupled to an exactly conserved neutron number operator. This is clear since $\mu$ appears in the same manner as a temporal gauge link. Another feature is that in the limit as $m_{N} \rightarrow \infty$, we find

$$
\operatorname{Tr} \exp \left[-\beta\left(H_{\bar{N} N}+H_{\bar{N} N \bar{N} N}\right)\right]=z_{0} \int D s D c D c^{*} \exp [-S]+O\left(m_{N}^{-2}\right) .
$$

Therefore any dependence on the temporal lattice spacing is suppressed by a factor of $m_{N}^{-2}$. This makes it possible to take the static neutron limit as a precision test of the simulation results. We have found this test quite useful in the process of code development and checking.

\section{DETERMINING COEFFICIENTS}

The interaction coefficient $C$ must be determined for various lattice spacings $a$ and $a_{t}$. We do this by summing all bubble chain diagrams contributing to neutron-neutron scattering as shown in Fig. 1.

The next step is to locate the pole in the scattering amplitude and compare with Lüscher's formula for energy levels in a finite periodic box [20, 21],

$$
E_{0}=\frac{4 \pi a_{\text {scatt }}}{m_{N} L^{3}}\left[1-c_{1} \frac{a_{\text {scatt }}}{L}+c_{2} \frac{a_{\text {scatt }}^{2}}{L^{2}}+\cdots\right],
$$

where $c_{1}=-2.837297, c_{2}=6.375183$. We then tune the coefficient $C$ to give the physically measured ${ }^{1} S_{0}$ scattering length. Since this scattering length is much larger than any other 


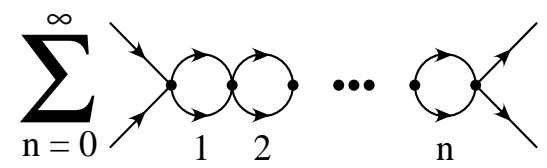

FIG. 1: Bubble chain diagrams contributing to neutron-neutron scattering.

length scale, we are in essence probing the universal behavior of interacting fermions at infinite scattering length. For our results we have used $a_{\text {scatt }}^{1} S_{0} \simeq-24 \mathrm{fm}$, though using the value of $-18 \mathrm{fm}$ specific for neutron-neutron scattering changes the operator coefficient by only $1 \%$.

The full bubble chain will have a pole when the amplitude for a single bubble times one vertex coefficient equals 1 . We take the center of mass frame and let the total incoming momentum of the two neutrons in physical units be

$$
\vec{p}_{\text {phys }}=\left(p_{* 0} a_{t}^{-1}, 0,0,0\right) .
$$

Since the physical pole occurs in Minkowski space, in the end $p_{0}$ will be imaginary. If we set $\mu=0$, then the amplitude for one bubble times one vertex coefficient is

$$
(1-6 h)^{2}\left(e^{-C \alpha_{t}}-1\right) B\left(p_{0}\right)
$$

where

$$
B\left(p_{0}\right)=\frac{1}{L^{3} L_{t}} \sum_{\vec{k}} \frac{1}{e^{m_{N} \alpha_{t}} e^{-i p_{* 0} / 2} e^{-i k_{* 0}}-1+\omega_{k}} \frac{1}{e^{m_{N} \alpha_{t}} e^{-i p_{* 0} / 2} e^{i k_{* 0}}-1+\omega_{k}},
$$

and the condition for the location of the pole is

$$
B\left(p_{0}\right)=\frac{1}{(1-6 h)^{2}\left(e^{-C \alpha_{t}}-1\right)} .
$$

By the definition of $\omega_{k}$ in (71) we see that $0 \leq \omega_{k} \leq 12 h$. We assume that the lattice spacing in the temporal direction is sufficiently small so that $h \leq \frac{1}{6}$. In practice this presents no problem since $m_{N}$ is quite large. We then have

$$
\begin{aligned}
0 & \leq \omega_{k} \leq 2, \\
-1 & \leq 1-\omega_{k} \leq 1 .
\end{aligned}
$$


We now make a variable transformation,

$$
z=e^{-i k_{* 0}}=e^{-i \frac{2 \pi}{L_{t}} k_{0}}
$$

We also take the zero temperature limit, $L_{t} \rightarrow \infty$, and convert from the discrete sum over $k_{0}$ to an integral clockwise over the unit circle in $z$ using

$$
\begin{gathered}
d z=-i \frac{2 \pi}{L_{t}} z d k_{0} \\
d k_{0}=i \frac{L_{t}}{2 \pi z} d z
\end{gathered}
$$

We then find

$$
\begin{aligned}
B\left(p_{0}\right) & =\frac{i}{2 \pi L^{3}} \sum_{k_{1}, k_{2}, k_{3}} \oint \frac{d z}{z\left(e^{m_{N} \alpha_{t}} e^{-i p_{* 0} / 2} z-1+\omega_{k}\right)\left(e^{m_{N} \alpha_{t}} e^{-i p_{* 0} / 2} z^{-1}-1+\omega_{k}\right)} \\
& =\frac{i}{2 \pi L^{3}} \sum_{k_{1}, k_{2}, k_{3}} \oint \frac{d z}{\left(e^{m_{N} \alpha_{t}} e^{-i p_{* 0} / 2} z-\left(1-\omega_{k}\right)\right)\left(e^{m_{N} \alpha_{t}} e^{-i p_{* 0} / 2}-\left(1-\omega_{k}\right) z\right)} \\
& =-\frac{i}{2 \pi L^{3}} \sum_{k_{1}, k_{2}, k_{3}} \oint \frac{e^{-m_{N} \alpha_{t}} e^{i p_{* 0} / 2}\left(1-\omega_{k}\right)^{-1} d z}{\left(z-e^{-m_{N} \alpha_{t}} e^{i p_{* 0} / 2}\left(1-\omega_{k}\right)\right)\left(z-e^{m_{N} \alpha_{t}} e^{-i p_{* 0} / 2}\left(1-\omega_{k}\right)^{-1}\right)} .
\end{aligned}
$$

When $\operatorname{Re}\left(i p_{* 0} \alpha_{t}^{-1}\right)<2 m_{N}$ we pick up the residue at $e^{-m_{N} \alpha_{t}} e^{i p_{* 0} / 2}\left(1-\omega_{k}\right)$, and the amplitude is

$$
\begin{aligned}
B\left(p_{0}\right) & =-\frac{i}{2 \pi L^{3}} \sum_{k_{1}, k_{2}, k_{3}} \frac{-2 \pi i\left(1-\omega_{k}\right)^{-1}}{\left(1-\omega_{k}\right)-e^{2 m_{N} \alpha_{t}} e^{-i p_{* 0}}\left(1-\omega_{k}\right)^{-1}} \\
& =-\frac{i}{2 \pi L^{3}} \sum_{k_{1}, k_{2}, k_{3}} \frac{-2 \pi i}{\left(1-\omega_{k}\right)^{2}-e^{2 m_{N} \alpha_{t}} e^{-i p_{* 0}}} \\
& =\frac{1}{L^{3}} \sum_{k_{1}, k_{2}, k_{3}} \frac{1}{e^{2 m_{N} \alpha_{t}} e^{-i p_{* 0}}-1+2 \omega_{k}-\omega_{k}^{2}} .
\end{aligned}
$$

Since we are interested in imaginary $p_{* 0}$ we switch variables,

$$
\begin{gathered}
E+2 m_{N}=i p_{* 0} \alpha_{t}^{-1}, \\
e^{-i p_{* 0}}=e^{-\alpha_{t}\left(E+2 m_{N}\right)},
\end{gathered}
$$

where $E$ is the energy in Minkowski space, with rest energy excluded. Finally we get

$$
B(E)=\frac{1}{L^{3}} \sum_{k_{1}, k_{2}, k_{3}} \frac{1}{e^{-\alpha_{t} E}-1+2 \omega_{k}-\omega_{k}^{2}},
$$


and the pole in the bubble chain sum occurs when

$$
B(E)=\frac{1}{(1-6 h)^{2}\left(e^{-C \alpha_{t}}-1\right)} .
$$

Using (24) we can determine $C$ for various spatial and temporal lattice spacings. Results for the lattice spacings used in the simulations presented here are shown in Table 2. We have also determined $\frac{d C}{d \alpha_{t}}$, which will be needed when computing the average energy by varying $\beta$ with fixed $L_{t}$.

Table 2: Contact potential coefficients $\left(\mathrm{MeV}^{-2}\right)$

\begin{tabular}{|l|l|l|l|}
\hline$a^{-1}(\mathrm{MeV})$ & $a_{t}^{-1}(\mathrm{MeV})$ & $C\left(\mathrm{MeV}^{-2}\right)$ & $\frac{d C}{d \alpha_{t}}$ \\
\hline 50 & 24 & $-11.6 \times 10^{-5}$ & $-2.21 \times 10^{-5}$ \\
\hline 60 & 32 & $-10.1 \times 10^{-5}$ & $-2.31 \times 10^{-5}$ \\
\hline 60 & 48 & $-8.75 \times 10^{-5}$ & $-1.91 \times 10^{-5}$ \\
\hline 70 & 64 & $-7.58 \times 10^{-5}$ & $-1.92 \times 10^{-5}$ \\
\hline 80 & 72 & $-6.96 \times 10^{-5}$ & $-2.04 \times 10^{-5}$ \\
\hline
\end{tabular}

\section{BUBBLE CHAIN SUMMATION}

In this section we discuss a simple semi-analytic calculation that we use to compare with the results of our simulations. At $T=0$ and if $k_{F}\left|a_{n n}\right|$ small the energy and particle densities can be calculated as an expansion in $k_{F}\left|a_{n n}\right|$. If the scattering length $a_{n n}$ is small this is equivalent to a perturbative expansion in the coupling constant $C$. If $a_{n n}$ is not small then an infinite set of particle-particle bubbles has to be summed. This is particularly obvious in the lattice cutoff scheme employed in this work. Since the coupling constant $C$ is fixed by matching the particle-particle bubble sum to the experimental scattering length at a given lattice spacing, a perturbative expansion of the equation of state in powers of $C$ will not be cutoff independent. An approximation scheme that will reproduce the lowest order $k_{F} a_{n n}$ expansion is the bubble chain summation shown in Figs. 2 and 3 .

The problem at $T=0$ is that the scattering length is very large, and the expansion in $k_{F}\left|a_{n n}\right|$ is not useful unless the density is extremely small, $\rho<10^{-4} \rho_{N}$. When $k_{F}\left|a_{n n}\right|$ is not small then corrections must be summed to all orders, and it is not obvious that there is any subset of diagrams that can approximate the full non-perturbative result. We note, however, that the bubble chain diagrams contain as a subset the diagrams with the minimum number of hole lines. These diagrams are summed by the low density hole line expansion. 


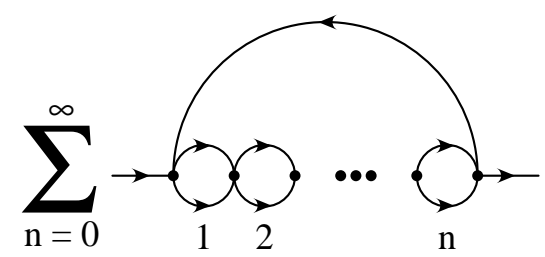

FIG. 2: Bubble chain diagrams contributing to the neutron self-energy.

The situation is simpler if the temperature $T$ is large compared to the degeneracy temperature $T_{F}=\left(3 \pi^{2} \rho\right)^{2 / 3} /(2 m)$. In this case a new length scale, the thermal wavelength or localization length appears

$$
\lambda_{T} \sim \sqrt{\frac{1}{2 m_{N} T}} .
$$

This length scale acts as an infrared regulator, cutting off long-distance correlations beyond this scale. In particular, it regulates the neutron-neutron scattering amplitude near threshold by giving the function $B(E)$ in (38) a correction of order $O\left(\lambda_{T}^{-1}\right)$. The net effect is that neutrons now have an effective scattering length of

$$
\left|a_{e f f}\right| \sim \min \left(\left|a_{n n}\right|, \lambda_{T}\right) .
$$

The expansion in $a_{e f f}^{3} \rho$ converges as long as $a_{e f f}^{3} \rho<1$ which is equivalent to $T>T_{F}$. In the following we compute the bubble chain diagrams shown in Figs. 2 and 3 ,

The bubble chain diagrams in the neutron self-energy form a geometric series. The sum is given by

$$
\Sigma(\vec{q})=-(1-6 h)^{2}\left(e^{-C \alpha_{t}}-1\right) \sum_{\vec{p}} \frac{D^{\text {free }}(\vec{p}-\vec{q})}{1-(1-6 h)^{2}\left(e^{-C \alpha_{t}}-1\right) B(\vec{p}, \mu)}
$$

where

$$
B(\vec{p}, \mu)=\frac{1}{L^{3} L_{t}} \sum_{\vec{k}} \frac{1}{e^{\left(m_{N}-\mu\right) \alpha_{t}} e^{-i p_{* 0} / 2} e^{-i k_{* 0}}-1+\omega_{p / 2+k}} \frac{1}{e^{\left(m_{N}-\mu\right) \alpha_{t}} e^{-i p_{* 0} / 2} e^{i k_{* 0}}-1+\omega_{-p / 2+k}} .
$$

We use this to compute the full neutron propagator

$$
D^{\text {full }}(\vec{q})=\frac{D^{\text {free }}(\vec{q})}{1-\Sigma(\vec{q}) D^{\text {free }}(\vec{q})},
$$

and the average number of neutrons is

$$
A=\frac{1}{\beta} \frac{\partial}{\partial \mu} \ln Z_{G}=2 L^{3}\left[1-\frac{e^{\left(m_{N}-\mu\right) \alpha_{t}}}{L_{t} L^{3}} \sum_{\vec{k}} D^{f u l l}(\vec{k}) e^{-i k_{* 0}}\right] .
$$




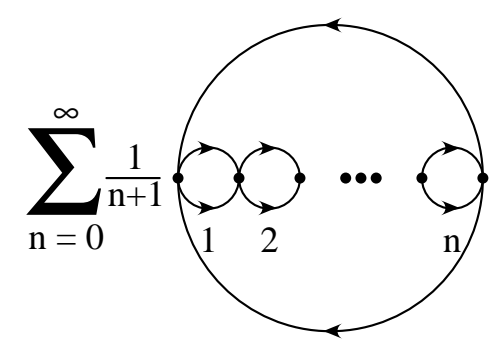

FIG. 3: Bubble chain diagrams contributing to the logarithm of the partition function.

In a similar fashion we compute the contribution of bubble chain diagrams to the logarithm of the partition function. The relevant diagrams are shown in Fig. 3, The factor of $\frac{1}{n+1}$ is the due to the cyclical symmetry of the diagram. We find

$$
\begin{aligned}
\ln Z_{G} & =\ln Z_{G}^{\text {free }} \\
& +\frac{1}{L_{t} L^{3}} \sum_{\vec{p}, \vec{q}} \frac{-\ln \left[1-(1-6 h)^{2}\left(e^{-C \alpha_{t}}-1\right) B(\vec{p}+\vec{q}, \mu)\right] D^{\text {free }}(\vec{p}) D^{\text {free }}(\vec{q})}{B(\vec{p}+\vec{q}, \mu)} .
\end{aligned}
$$

From this we can compute the average energy $E$

$$
E=-\frac{\partial \ln Z_{G}}{\partial \beta}+\left(-m_{N}+\mu\right) A,
$$

where we have subtracted out the rest energy. The derivative with respect to $\beta$ is calculated at fixed $L_{t}$ by varying $\alpha_{t}$,

$$
E=-\frac{1}{L_{t}} \frac{\partial \ln Z_{G}}{\partial \alpha_{t}}+\left(-m_{N}+\mu\right) A .
$$

We must take into account the dependence of $C$ on $\alpha_{t}$, and $\frac{d C}{d \alpha_{t}}$ for various lattice spacings are shown in Table 2 .

\section{COMPUTATIONAL METHODS}

We use the hybrid Monte Carlo (HMC) algorithm [22] to generate field configurations. Roughly $10^{5}$ five-step HMC trajectories were run, split across 9 processors running completely independent trajectories. Averages and errors were computed by comparing the results of each processor. While the HMC algorithm has become standard in lattice QCD, it may not be so well known in the general nuclear theory community. We therefore include a brief overview of the method as applied to our simulation. 
We want to sample the partition function

$$
Z_{G} \propto \int D s D \psi D \psi^{*} \exp \left[-\psi_{i}^{*} Q_{i j}^{\prime}(s) \psi_{j}-V(s)\right]
$$

where the $s_{\alpha}$ 's are bosonic fields and the $\psi_{i}$ 's are fermionic fields. We use a prime since we will redefine $Q_{i j}^{\prime}$ shortly. We can rewrite this as a bosonic path integral

$$
Z_{G} \propto \int D s D \phi D \phi^{*} \exp \left[-S^{\prime}(\phi, s)\right]
$$

where

$$
S^{\prime}(\phi, s)=\phi_{i}^{*} Q_{i j}^{\prime-1}(s) \phi_{j}+V(s) .
$$

The $\phi_{i}$ 's are bosonic fields and are called pseudofermion fields. The partition function can be written as

$$
Z_{G} \propto \int D s D p D \phi D \phi^{*} \exp [-H(\phi, s, p)]
$$

where

$$
H(\phi, s, p)=S^{\prime}(\phi, s)+\frac{1}{2} p_{\alpha} p_{\alpha}
$$

We note that

$$
\begin{aligned}
\frac{\partial S^{\prime}(\phi, s)}{\partial s_{\alpha}} & =\phi_{i}^{*} \frac{\partial Q_{i j}^{\prime-1}(s)}{\partial s_{\alpha}} \phi_{j}+\frac{\partial V(s)}{\partial s_{\alpha}} \\
& =-\phi_{i}^{*} Q_{i j}^{\prime-1}(s) \frac{\partial Q_{j k}^{\prime}(s)}{\partial s_{\alpha}} Q_{k l}^{\prime-1}(s) \phi_{l}+\frac{\partial V(s)}{\partial s_{\alpha}} .
\end{aligned}
$$

In our case the determinant of $Q^{\prime}$ is real and non-negative. We can therefore replace $Q_{i j}^{\prime}$ by a positive semi-definite Hermitian matrix $Q_{i j}$, with the same determinant. In our case $Q_{i j}^{\prime}$ has the block diagonal structure

$$
Q^{\prime}=\left[\begin{array}{ll}
K & 0 \\
0 & K
\end{array}\right],
$$

one block for the up spins and one block for the down spins. Clearly $K_{i j}$ is a matrix with half the dimension of $Q_{i j}^{\prime}$. If we let

$$
Q=K^{\dagger} K
$$

then

$$
Q^{-1}=K^{-1}\left(K^{\dagger}\right)^{-1}
$$


and

$$
\operatorname{det} Q=\operatorname{det} Q^{\prime}
$$

So now instead of $S^{\prime}(\phi, s)$ we can use

$$
\begin{aligned}
S(\phi, s) & =\phi_{i}^{*} Q_{i j}^{-1}(s) \phi_{j}+V(s) \\
& =\xi_{j}^{*}(s) \xi_{j}(s)+V(s)
\end{aligned}
$$

where

$$
\begin{aligned}
\xi_{i} & =\left(K^{\dagger}\right)_{i j}^{-1} \phi_{j}, \\
\phi_{i} & =\left(K^{\dagger}\right)_{i j} \xi_{j} .
\end{aligned}
$$

When we compute the derivative with respect to $s_{\alpha}$, we have

$$
\frac{\partial}{\partial s_{\alpha}}\left[K^{-1}\left(K^{\dagger}\right)^{-1}\right]=-K^{-1} \frac{\partial K}{\partial s_{\alpha}} K^{-1}\left(K^{\dagger}\right)^{-1}-K^{-1}\left(K^{\dagger}\right)^{-1} \frac{\partial K^{\dagger}}{\partial s_{\alpha}}\left(K^{\dagger}\right)^{-1}
$$

Let us define

$$
\eta_{i}=K_{i j}^{-1} \xi_{j}
$$

Then

$$
\phi_{i}^{*} \frac{\partial}{\partial s_{\alpha}}\left[K^{-1}\left(K^{\dagger}\right)^{-1}\right]_{i j} \phi_{j}=-\xi_{i}^{*}\left[\frac{\partial K}{\partial s_{\alpha}}\right]_{i j} \eta_{j}-\eta_{i}^{*}\left[\frac{\partial K^{\dagger}}{\partial s_{\alpha}}\right]_{i j} \xi_{j} .
$$

Therefore

$$
\frac{\partial S(\phi, s)}{\partial s_{\alpha}}=-\xi_{i}^{*}\left[\frac{\partial K}{\partial s_{\alpha}}\right]_{i j} \eta_{j}-\eta_{i}^{*}\left[\frac{\partial K^{\dagger}}{\partial s_{\alpha}}\right]_{i j} \xi_{j}+\frac{\partial V(s)}{\partial s_{\alpha}} .
$$

The steps for the HMC algorithm are now as follows.

Step 1: Select an arbitrary initial real-valued configuration $s_{\alpha}^{0}$.

Step 2: Select a complex-valued configuration $\xi_{j}$ according to the Gaussian random distribution,

$$
P\left(\xi_{j}\right) \propto \exp \left[-\left|\xi_{j}\right|^{2}\right]
$$

and let

$$
\begin{aligned}
\phi_{i} & =\left(K^{\dagger}\right)_{i j} \xi_{j}, \\
\eta_{i} & =K_{i j}^{-1} \xi_{j} .
\end{aligned}
$$


Step 3: Select real-valued $p_{\alpha}^{0}$ according to the Gaussian random distribution

$$
P\left(p_{\alpha}^{0}\right) \propto \exp \left[-\frac{1}{2}\left(p_{\alpha}^{0}\right)^{2}\right] .
$$

Step 4: Let

$$
s_{\alpha}(0)=s_{\alpha}^{0}
$$

and

$$
\tilde{p}_{\alpha}(0)=p_{\alpha}^{0}-\frac{\varepsilon}{2}\left[-\xi_{i}^{*}\left[\frac{\partial K}{\partial s_{\alpha}}\right]_{i j} \eta_{j}-\eta_{i}^{*}\left[\frac{\partial K^{\dagger}}{\partial s_{\alpha}}\right]_{i j} \xi_{j}+\frac{\partial V(s)}{\partial s_{\alpha}}\right]_{s=s^{0}},
$$

for some small positive $\varepsilon$.

Step 5: For $n=0,1, \ldots, N-1$, let

$$
\begin{aligned}
& s_{\alpha}(n+1)=s_{\alpha}(n)+\varepsilon \tilde{p}_{\alpha}(n) \\
& \tilde{p}_{\alpha}(n+1)=\tilde{p}_{\alpha}(n)-\varepsilon\left[-\xi_{i}^{*}\left[\frac{\partial K}{\partial s_{\alpha}}\right]_{i j} \eta_{j}-\eta_{i}^{*}\left[\frac{\partial K^{\dagger}}{\partial s_{\alpha}}\right]_{i j} \xi_{j}+\frac{\partial V(s)}{\partial s_{\alpha}}\right]_{s=s(n+1)} .
\end{aligned}
$$

Step 6: Let

$$
p_{\alpha}(N)=\tilde{p}_{\alpha}(N)+\frac{\varepsilon}{2}\left[-\xi_{i}^{*}\left[\frac{\partial K}{\partial s_{\alpha}}\right]_{i j} \eta_{j}-\eta_{i}^{*}\left[\frac{\partial K^{\dagger}}{\partial s_{\alpha}}\right]_{i j} \xi_{j}+\frac{\partial V(s)}{\partial s_{\alpha}}\right]_{s=s(N)} .
$$

Step 7: Select a random number $r \in[0,1)$. If

$$
r<\exp \left[-H(\phi, s(N), p(N))+H\left(\phi, s^{0}, p^{0}\right)\right]
$$

then let

$$
s^{0}=s(N) .
$$

Otherwise leave $s^{0}$ as is. In either case go back to Step 2.

The total number of neutrons, $A$, is

$$
\begin{aligned}
A & =\frac{1}{\beta} \frac{\partial}{\partial \mu} \ln Z_{G}=2 L^{3}-\frac{1}{\beta} \frac{\int D s D c^{\prime} D c^{*} \frac{\partial S}{\partial \mu} \exp [-S]}{\int D s D c^{\prime} D c^{*} \exp [-S]} \\
& =2 L^{3}\left[1-\frac{e^{\left(m_{N}-\mu\right) \alpha_{t}} \int D s D c^{\prime} D c^{*} c_{\uparrow}^{\prime}(\vec{n}+\hat{0}) c_{\uparrow}^{*}(\vec{n}) \exp [-S]}{\int D s D c^{\prime} D c^{*} \exp [-S]}\right] .
\end{aligned}
$$


for any lattice site $\vec{n}$. Dividing by the volume $V=L^{3}$ gives the density $\rho$ in lattice units. We compute the total energy using (48),

$$
\begin{aligned}
E & =-\frac{1}{L_{t}} \frac{\partial \ln Z_{G}}{\partial \alpha_{t}}+\left(-m_{N}+\mu\right) A \\
& =\frac{1}{L_{t}} \frac{\int D s D c^{\prime} D c^{*} \frac{\partial S}{\partial \alpha_{t}} \exp [-S]}{\int D s D c^{\prime} D c^{*} \exp [-S]}+\left(-m_{N}+\mu\right) A,
\end{aligned}
$$

where we take into account the $\alpha_{t}$ dependence of $C$ when computing $\frac{\partial S}{\partial \alpha_{t}}$. We then have

$$
E=2 L^{3} \frac{\int D s D c^{\prime} D c^{*} f\left(s, c^{\prime}, c^{*}\right) \exp [-S]}{\int D s D c^{\prime} D c^{*} \exp [-S]}+\left(-m_{N}+\mu\right) A
$$

where

$$
\begin{aligned}
f\left(s, c^{\prime}, c^{*}\right) & =-\left(m_{N}-\mu\right) e^{\left(m_{N}-\mu\right) \alpha_{t}} c_{\uparrow}^{\prime}(\vec{n}+\hat{0}) c_{\uparrow}^{*}(\vec{n}) \\
& +\frac{\partial}{\partial \alpha_{t}}\left(e^{\sqrt{-C \alpha_{t}} s(\vec{n})+\frac{C \alpha_{t}}{2}}(1-6 h)\right) c_{\uparrow}^{\prime}(\vec{n}) c_{\uparrow}^{*}(\vec{n}) \\
& +\frac{1}{2 m_{N}} \sum_{l_{s}}\left[c_{\uparrow}^{\prime}\left(\vec{n}+\hat{l}_{s}\right) c_{\uparrow}^{*}(\vec{n})+c_{\uparrow}^{\prime}\left(\vec{n}-\hat{l}_{s}\right) c_{\uparrow}^{*}(\vec{n})\right]
\end{aligned}
$$

for any lattice site $\vec{n}$.

\section{FREE NEUTRON RESULTS}

To better understand our lattice discretization errors, we compare our free neutron results on the lattice with the continuum free Fermi gas. For a continuum free Fermi gas, the logarithm of the partition function is

$$
\ln Z_{G}^{\text {free }}=\ln Z_{G, \uparrow}^{\text {free }}+\ln Z_{G, \downarrow}^{\text {free }}=2 \ln Z_{G, \uparrow}^{\text {free }},
$$

where the logarithm of the single spin partition function is

$$
\begin{aligned}
\ln Z_{\uparrow}^{\text {free }} & =V \int \frac{d^{3} \vec{p}}{(2 \pi)^{3}} \ln \left[1+e^{-\beta\left(\frac{\vec{p}^{2}}{2 m_{N}}+m_{N}-\mu\right)}\right] \\
& =\frac{V}{2 \pi^{2}} \int_{0}^{\infty} d p p^{2} \ln \left[1+e^{-\beta\left(\frac{p^{2}}{2 m_{N}}+m_{N}-\mu\right)}\right] .
\end{aligned}
$$

Therefore the energy density is

$$
\frac{E_{\uparrow}^{\text {free }}}{V}=\frac{1}{2 \pi^{2}} \int_{0}^{\infty} d p \frac{p^{4}}{2 m_{N}} \frac{1}{e^{\beta\left(\frac{p^{2}}{2 m_{N}}+m_{N}-\mu\right)}+1},
$$




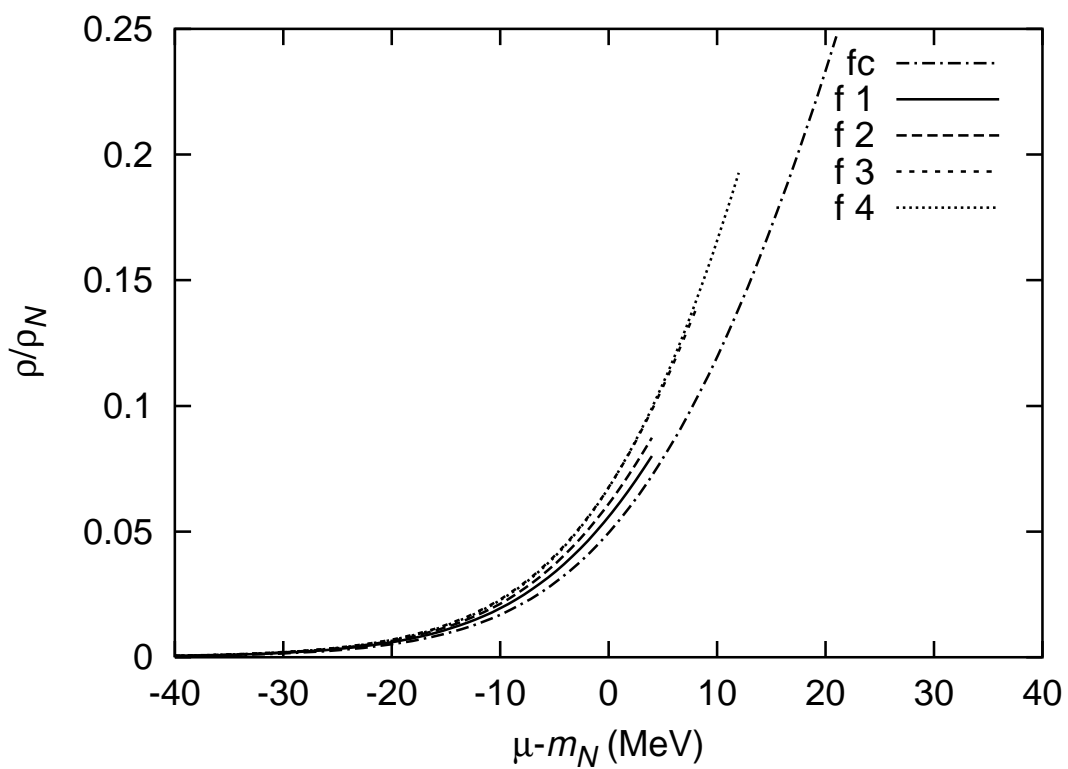

FIG. 4: Density versus chemical potential for free neutrons on the lattice at $T=8 \mathrm{MeV}$ and various lattice spacings. The curves labeled f1-f4 refer to the lattice spacings defined in Table 1. The curve labeled fc shows the continuum limit for free neutrons.

and the number density is

$$
\rho_{\uparrow}^{\text {free }}=\frac{A^{\text {free }}}{V}=\frac{1}{2 \pi^{2}} \int_{0}^{\infty} d p p^{2} \frac{1}{e^{\beta\left(\frac{p^{2}}{2 m_{N}}+m_{N}-\mu\right)}+1} .
$$

We double these to get the results for both spins. In the limit as $\rho^{\text {free }} \rightarrow 0$ we find the usual equipartition result for the energy per neutron,

$$
\frac{E^{\text {free }}}{A^{\text {free }}}=\frac{3}{2} T
$$

A plot of density versus chemical potential at temperature $T=8 \mathrm{MeV}$ is shown in Fig. 4. The energy per neutron at temperature $T=8 \mathrm{MeV}$ is shown in Fig. 5. In order to avoid large cutoff effects, we only present results at densities corresponding with lattice fillings of about one-quarter or less. This is why our data at longer lattice spacings terminates at lower densities.

We see in Figs. 4 and 5 some residual dependence on lattice spacings. While it is a small effect, it does make it visually confusing to overlay plots for different lattice spacings. We mentioned the possibility of using improved actions to reduce residual lattice discretization error. In this analysis, however, we use a less expensive route. We will simply rescale our 


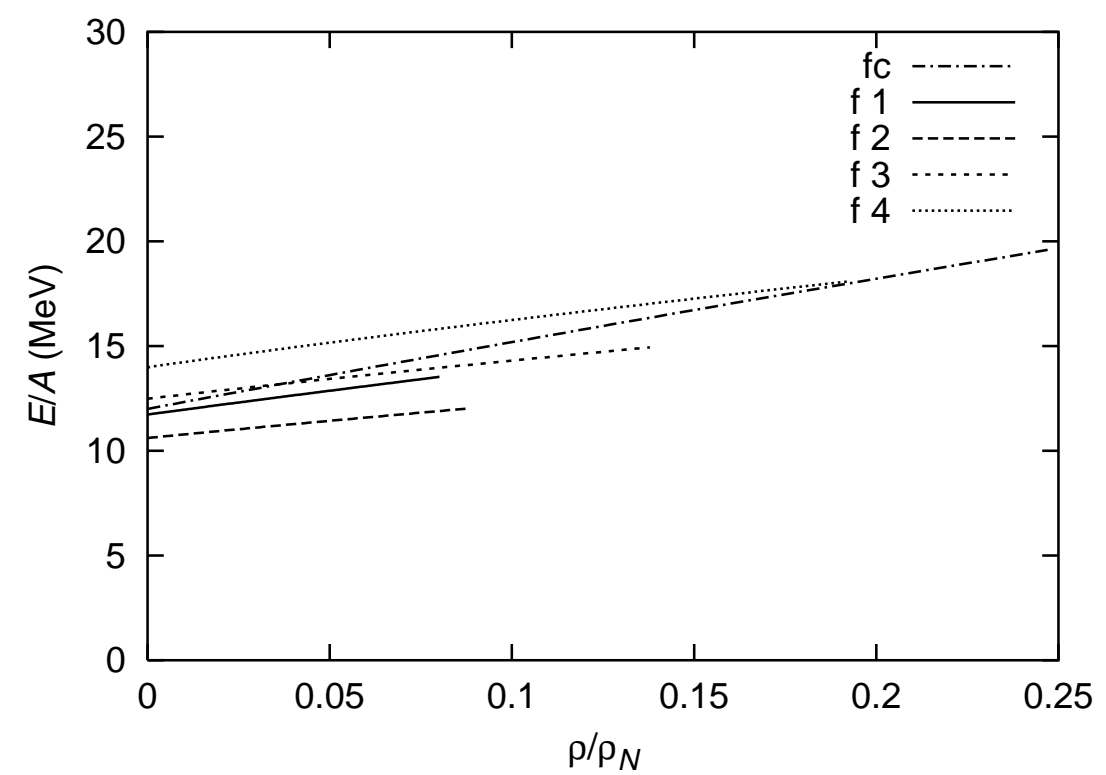

FIG. 5: Energy per neutron versus density for free neutrons on the lattice at $T=8 \mathrm{MeV}$ and various lattice spacings.

densities and energies so that the free lattice results and free continuum results agree at $\mu=m_{N}$,

$$
\begin{array}{r}
\rho\left(a, a_{t}, L, T, \mu\right) \rightarrow \rho\left(a, a_{t}, L, T, \mu\right) \cdot \frac{\rho^{\text {free }}\left(a=0, a_{t}=0, L=\infty, T, \mu=m_{N}\right)}{\rho^{\text {free }}\left(a, a_{t}, L, T, \mu=m_{N}\right)}, \\
E\left(a, a_{t}, L, T, \mu\right) \rightarrow E\left(a, a_{t}, L, T, \mu\right) \cdot \frac{E^{\text {free }}\left(a=0, a_{t}=0, L=\infty, T, \mu=m_{N}\right)}{E^{\text {free }}\left(a, a_{t}, L, T, \mu=m_{N}\right)} .
\end{array}
$$

We will apply the same multiplicative adjustment to all lattice results. This includes free lattice results, bubble chain diagram results, and lattice simulation results.

\section{VOLUME DEPENDENCE}

For the $T=8 \mathrm{MeV}$ simulations we use a lattice volume of $(13 \mathrm{fm})^{3}$ or larger. For the $T=4 \mathrm{MeV}$ simulations we use a lattice volume of $(20 \mathrm{fm})^{3}$ or larger. The dimensions of our $L^{3} \times L_{t}$ lattices are shown in Tables 3 and 4 . 
Table 3: Lattice dimensions for $T=8 \mathrm{MeV}$

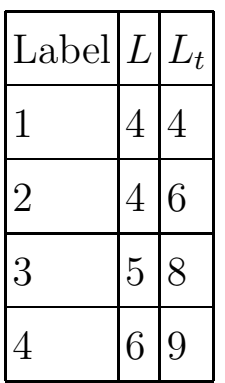

Table 4: Lattice dimensions for $T=4 \mathrm{MeV}$

\begin{tabular}{|l|l|l|}
\hline Label & $L$ & $L_{t}$ \\
\hline 0 & 5 & 6 \\
\hline 1 & 6 & 8 \\
\hline
\end{tabular}

We have run simulations at both smaller and larger volumes. In Table 5 we show the results for $T=8 \mathrm{MeV}$ and $\mu-m_{N}=-2 \mathrm{MeV}, a^{-1}=60 \mathrm{MeV}$, and $a_{t}^{-1}=32 \mathrm{MeV}$. Since we are not changing the lattice spacings for this comparison we can compare raw data without rescaling $\rho$ and $E$.

Table 5: $L$ dependence for $T=8 \mathrm{MeV}$

\begin{tabular}{|l|l|l|l|l|l|l|}
\hline$L$ & $\frac{\rho^{\text {free }}}{\rho_{N}}$ & $\frac{E^{\text {free }}}{A^{\text {free }}}(\mathrm{MeV})$ & $\frac{\rho^{\text {bubble }}}{\rho_{N}}$ & $\frac{E^{\text {bubble }}}{A^{\text {bubble }}}(\mathrm{MeV})$ & $\frac{\rho^{\text {simulation }}}{\rho_{N}}$ & $\frac{E^{\text {simulation }}}{A^{\text {simulation }}}(\mathrm{MeV})$ \\
\hline 3 & 0.04588 & 12.582 & 0.08176 & 6.514 & $0.0885(4)$ & $6.19(2)$ \\
\hline 4 & 0.04596 & 12.777 & 0.08215 & 6.539 & $0.0890(2)$ & $6.21(2)$ \\
\hline 5 & 0.04600 & 12.757 & 0.08217 & 6.531 & $0.0886(3)$ & $6.19(2)$ \\
\hline 6 & 0.04600 & 12.756 & 0.08217 & 6.531 & $0.0891(3)$ & $6.20(2)$ \\
\hline
\end{tabular}

In Table 6 we show analogous results for $T=4 \mathrm{MeV}$ and $\mu=m_{N}, a^{-1}=50 \mathrm{MeV}$, and $a_{t}^{-1}=24 \mathrm{MeV}$.

Table 6: $L$ dependence for $T=4 \mathrm{MeV}$

\begin{tabular}{|l|l|l|l|l|l|l|}
\hline$L$ & $\frac{\rho^{\text {free }}}{\rho_{N}}$ & $\frac{E^{\text {free }}}{A^{\text {free }}}(\mathrm{MeV})$ & $\frac{\rho^{\text {bubble }}}{\rho_{N}}$ & $\frac{E^{\text {bubble }}}{A^{\text {bubble }}}(\mathrm{MeV})$ & $\frac{\rho^{\text {simulation }}}{\rho_{N}}$ & $\frac{E^{\text {simulation }}}{A^{\text {simulation }}}(\mathrm{MeV})$ \\
\hline 4 & 0.02234 & 7.349 & 0.04663 & 3.474 & $0.0536(3)$ & $3.33(2)$ \\
\hline 5 & 0.02238 & 7.344 & 0.04667 & 3.469 & $0.0533(2)$ & $3.33(2)$ \\
\hline 6 & 0.02238 & 7.341 & 0.04666 & 3.469 & $0.0530(2)$ & $3.35(2)$ \\
\hline 7 & 0.02238 & 7.341 & 0.04666 & 3.469 & $0.0530(2)$ & $3.35(2)$ \\
\hline
\end{tabular}

These results suggest that finite volume effects for the lattice sizes listed in Tables 3 and 4 are smaller than our statistical errors. If we take the volume dependence from the free and bubble chain calculations as a guide, then the finite volume errors are well below the $1 \%$ level. 


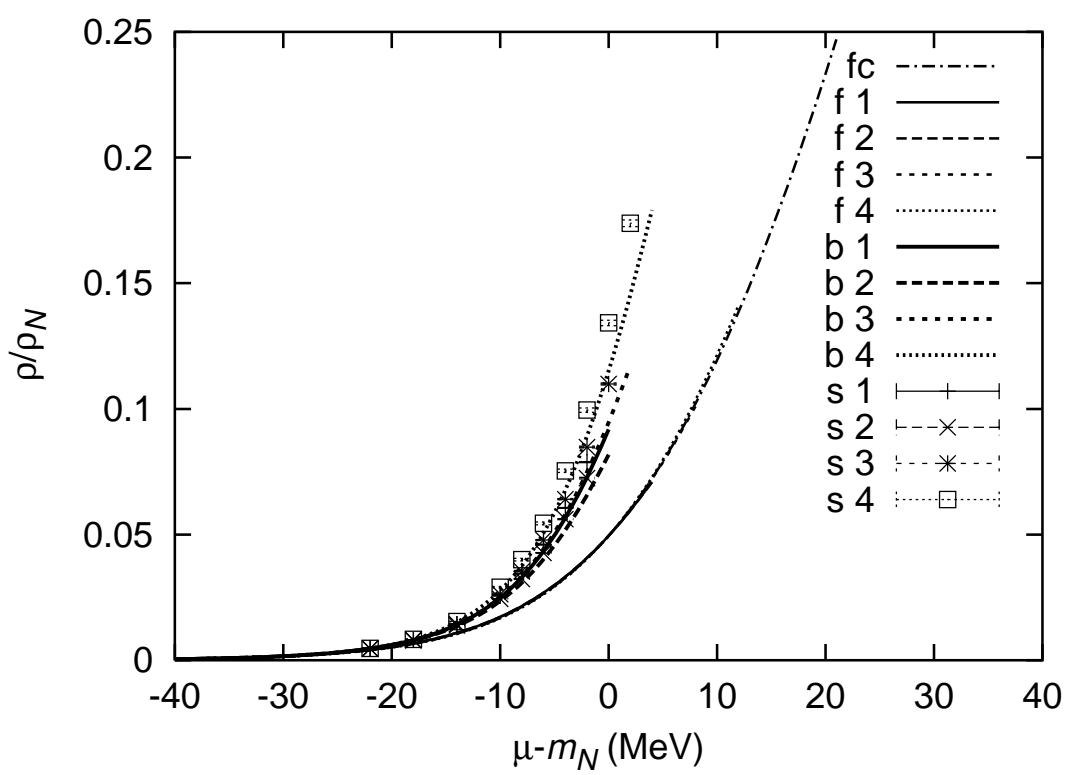

FIG. 6: Density versus chemical potential at $T=8 \mathrm{MeV}$ and various lattice spacings. The curves labeled f1-f4 show free neutron results, b1-b4 show the bubble chain results, and s1-s4 show numerical simulations. The corresponding lattice spacings are given in Table 1.

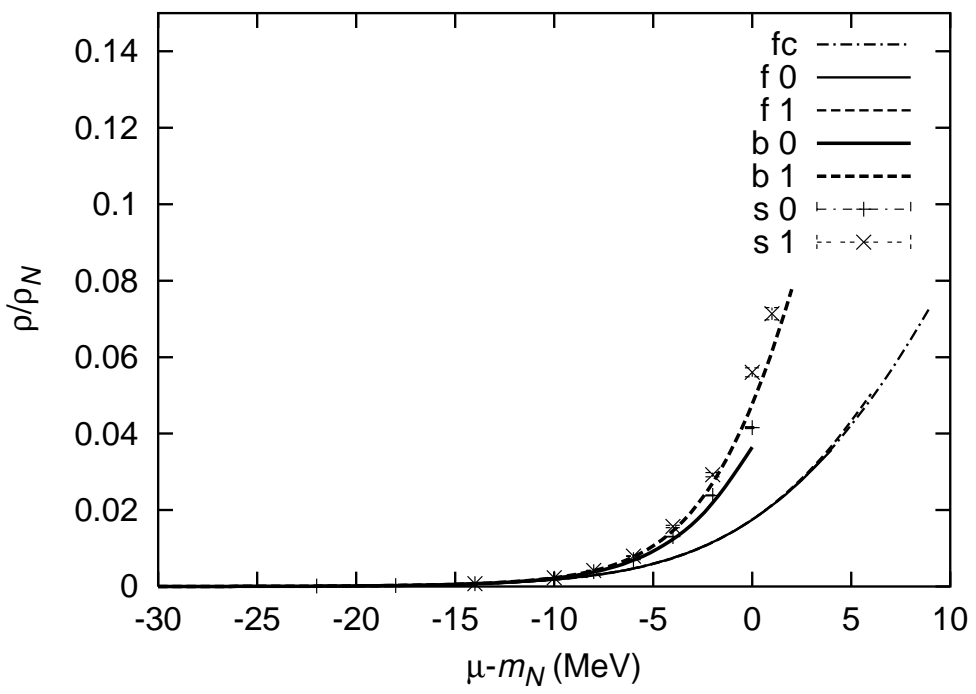

FIG. 7: Density versus chemical potential at $T=4 \mathrm{MeV}$ and various lattice spacings.

\section{DENSITY VERSUS CHEMICAL POTENTIAL}

In Fig. [6] we plot density versus chemical potential for $T=8 \mathrm{MeV}$, and in Fig. [7] we plot density versus chemical potential for $T=4 \mathrm{MeV}$. In both cases we see agreement among 


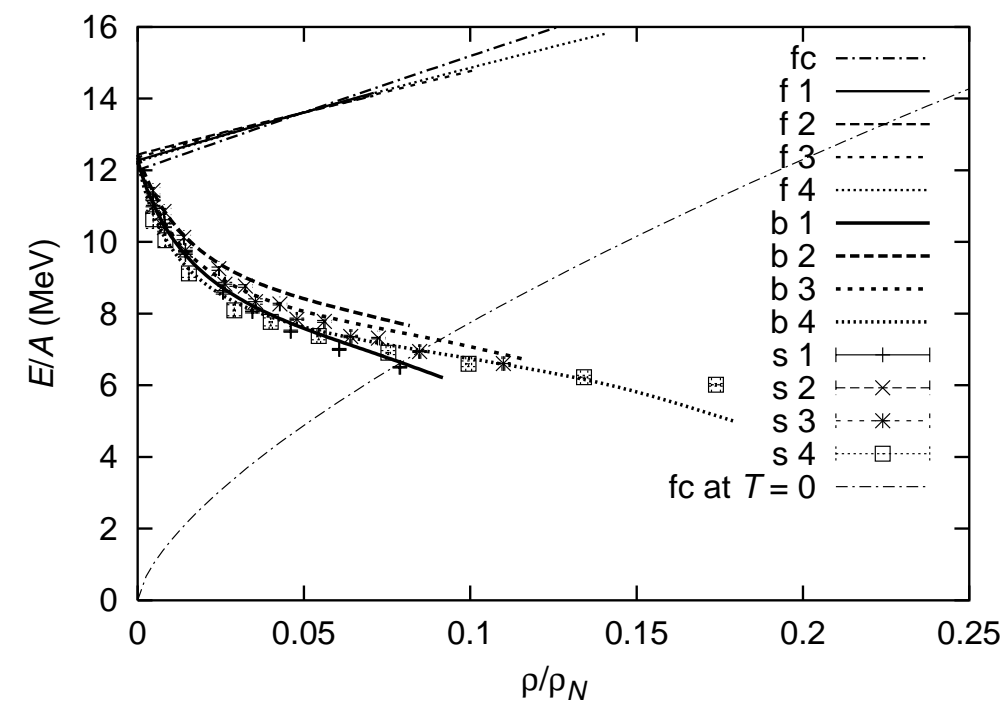

FIG. 8: Energy per neutron versus density at $T=8 \mathrm{MeV}$ for various lattice spacings.

data for different lattice spacings. This suggests that we have properly renormalized the interaction and absorbed the lattice spacing dependence into the scale dependent interaction coefficient. We observe no phase transitions as a function of chemical potential. We note, in particular, that we can choose the chemical potential such that the occupation number in the interacting theory remains small. This implies that there is no instability towards a fully occupied ground state. As expected for a theory with attractive interactions the density at a given chemical potential is larger in the interacting theory. We observe that this behavior is well described by the bubble chain results for $T>T_{F}$, the low-density regime where we expect agreement.

\section{ENERGY PER NEUTRON VERSUS DENSITY}

In Fig. 8 we plot energy per neutron versus density for $T=8 \mathrm{MeV}$, and in Fig. 9 we plot energy per neutron versus density for $T=4 \mathrm{MeV}$. In both cases we see good agreement among data for different lattice spacings. As expected, the energy per particle approaches $1.5 T$ in the dilute limit. The energy per particle decreases as a function of density in the regime that we have studied. At small $\rho$ the slope is very steep, which is consistent with the perturbative result for particles that have a large negative scattering length. We note, however, that the behavior is not linear, even at very small density. The simulations are 


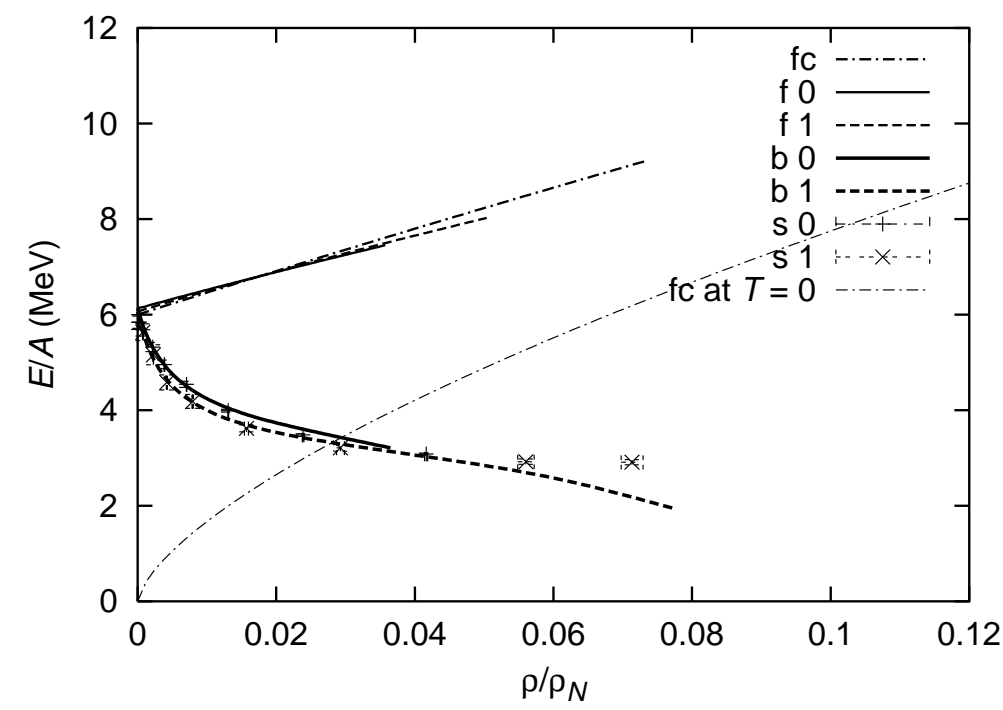

FIG. 9: Energy per neutron versus density at $T=4 \mathrm{MeV}$ for various lattice spacings.

very well described by the bubble chain results for $T>T_{F}$. We also observe that for the larger densities, $\rho>0.1 \rho_{N}$ at $T=8 \mathrm{MeV}$ and $\rho>0.03 \rho_{N}$ at $T=4 \mathrm{MeV}$, the energy per particle is smaller than the result for a free neutron gas at zero temperature. In this regime the Fermi energy of the degenerate system is lower than the temperature. Our results suggest that the parameter $\xi$ defined in (11) is smaller than 0.5. We should note, however, that the parameter $k_{F} r_{n n} \sim k_{F} a$, where $a$ is the lattice spacing, is of order 1 and simulations at lower temperature will be required in order to make more definitive estimates of $\xi$.

The decrease in the energy per neutron with increasing density does not necessarily imply an instability to neutron clustering. At nonzero temperature entropy must also be taken into account, and the question of whether or not phase separation occurs will be resolved in the next section when we look at the equation of state.

\section{EQUATION OF STATE}

We integrate the density as a function of chemical potential to measure the pressure,

$$
P=\frac{T}{V} \ln Z_{G}=\frac{1}{V} \int_{-\infty}^{\mu} A\left(\mu^{\prime}\right) d \mu^{\prime}=\int_{-\infty}^{\mu} \rho\left(\mu^{\prime}\right) d \mu^{\prime} .
$$

We perform the integration by least-squares fitting $\rho\left(\mu^{\prime}\right)$ with a function of the form

$$
\rho\left(\mu^{\prime}\right)=\left(c_{0}+c_{1} \mu^{\prime}+c_{2} \mu^{\prime 2}\right) \exp \left(b \mu^{\prime}\right) .
$$




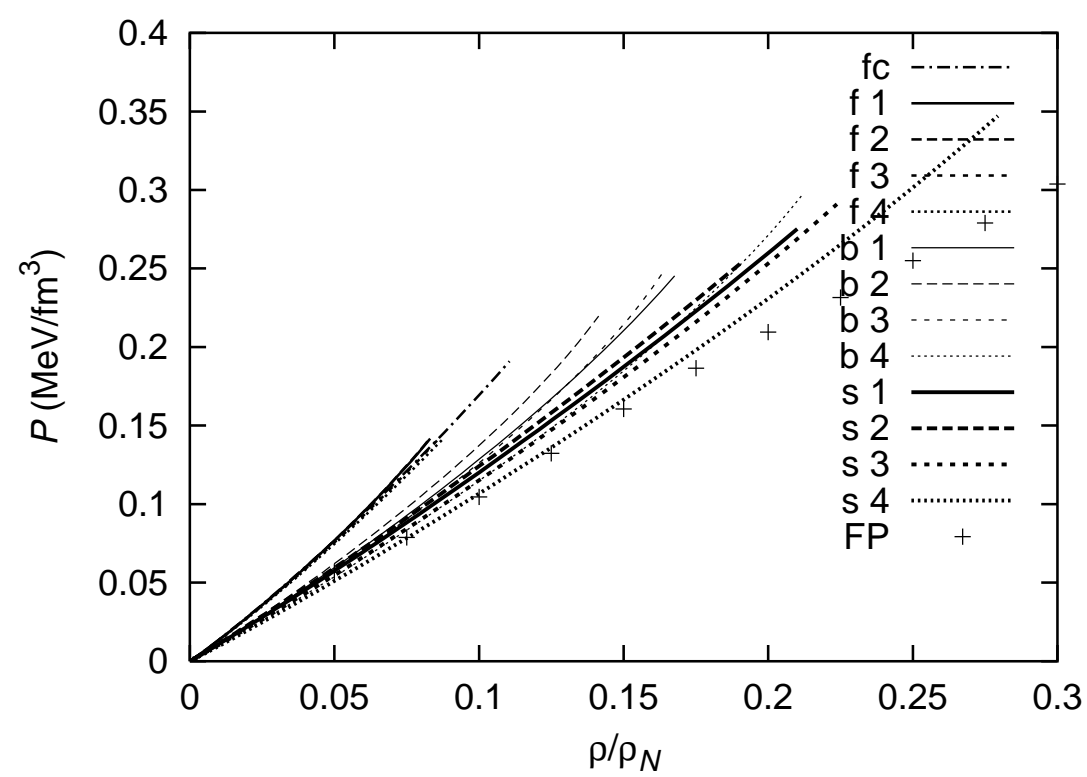

FIG. 10: Pressure versus density at $T=8 \mathrm{MeV}$ for various lattice spacings. The crosses show the results of a variational calculation by Friedman and Pandharipande [9].

We can then perform the integration analytically. In Fig. 10 we plot the pressure versus density for $T=8 \mathrm{MeV}$, and in Fig. 11 we plot the pressure versus density for $T=4 \mathrm{MeV}$. In both cases the pressure is a smooth strictly increasing function of density. Therefore we conclude that there is no indication of phase separation.

There are many models we could use to compare with our results. We first consider the results of a variational calculation by Friedman and Pandharipande [9]. They use a realistic Hamiltonian that consists of the Argonne $v_{14}$ interaction supplemented by a threebody force. We have taken the data for different temperatures given in Table 4 of [9] and interpolated to obtain the pressure for $T=4 \mathrm{MeV}$ and $T=8 \mathrm{MeV}$. The result are shown by the crosses in Figs. 10 and 11. We observe that the agreement with our calculations is remarkably good. There are a number of factors that are likely to contribute to this result. One is the fact that for the low densities considered in the present work explicit pions as well as three-body forces are not important. Another point is that we work with relatively coarse lattices. On these lattices the lattice spacing is close to the effective range parameter in neutron-neutron scattering.

We also show the equation of state for a simple phenomenological model of the equation of state described in 23] and the review article [24]. The model contains a parameterization 


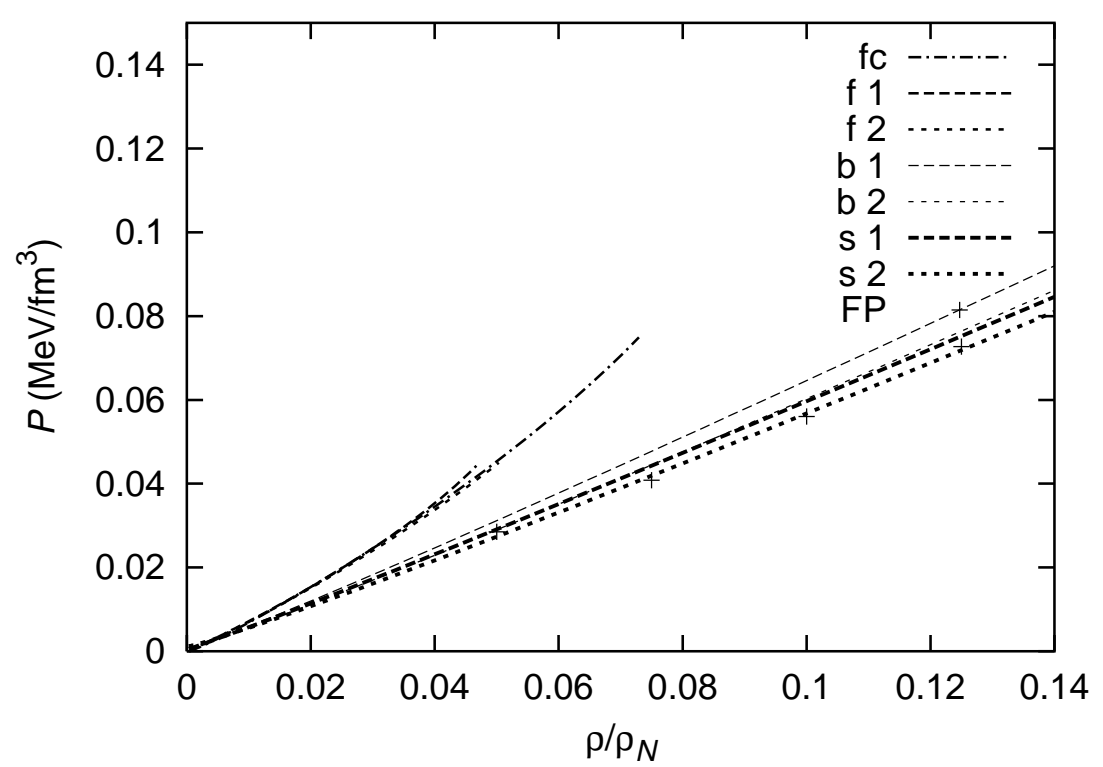

FIG. 11: Pressure versus density at $T=4 \mathrm{MeV}$ for various lattice spacings. The crosses show the results of a variational calculation by Friedman and Pandharipande [9].

of the equation of state of symmetric nuclear matter adjusted to the saturation properties and the compressibility. The authors consider three possible functional forms of asymmetry,

$$
\begin{array}{ll}
\text { (version 1) } & P_{a s y}=2 e_{a} \rho_{N}\left(\frac{\rho}{\rho_{N}}\right)^{3} \delta^{2}, \\
\text { (version 2) } & P_{a s y}=e_{a} \rho_{N}\left(\frac{\rho}{\rho_{N}}\right)^{2} \delta^{2}, \\
\text { (version 3) } & P_{a s y}=\frac{1}{2} e_{a} \rho_{N}\left(\frac{\rho}{\rho_{N}}\right)^{\frac{3}{2}} \delta^{2},
\end{array}
$$

where $e_{a} \simeq 20 \mathrm{MeV}$, and the asymmetry parameter $\delta$ is defined as

$$
\delta=\frac{\rho_{n}-\rho_{p}}{\rho_{n}+\rho_{p}}
$$

The equations of state of the three different models for $T=8 \mathrm{MeV}$ and $T=4 \mathrm{MeV}$ are shown in Figs. 12 and 13. For comparison, we also show the variational results of Friedman and Pandharipande. We observe that our results, as well as the results of Friedman and Pandharipande, appear to agree most closely with version 3. Further investigations are needed to determine whether other properties of this simple model agree with our lattice simulation results. 


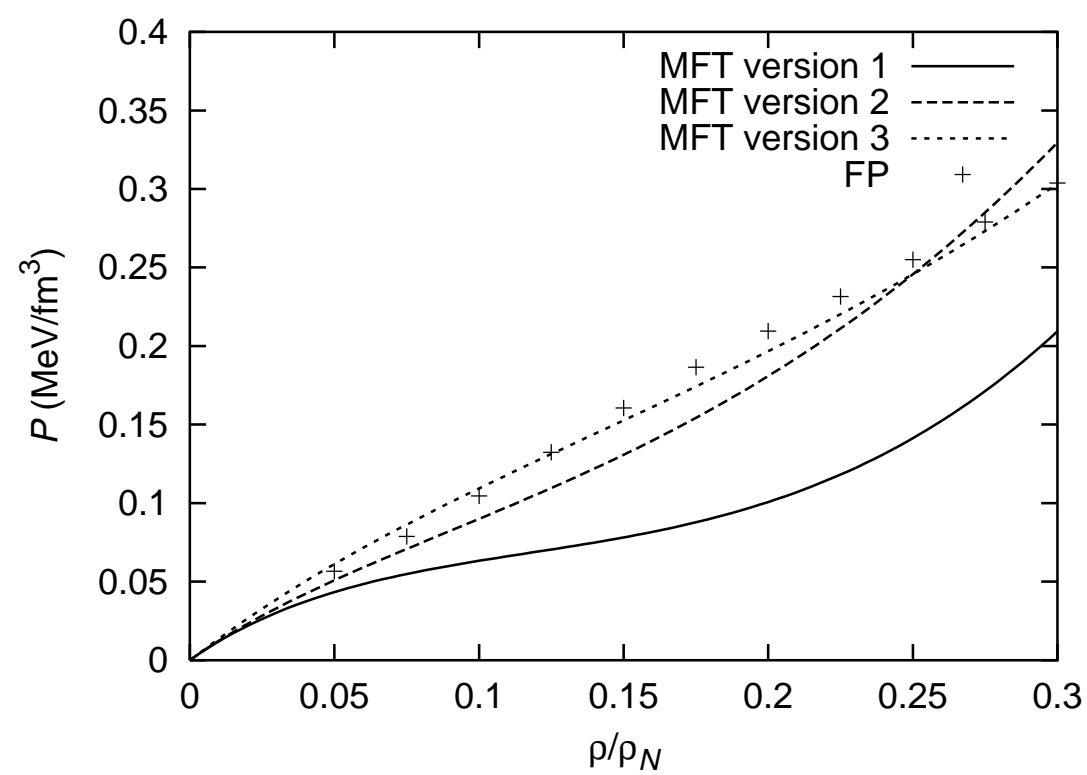

FIG. 12: Pressure versus density at $T=8 \mathrm{MeV}$ for the phenomenological equation of state discussed in 23]. The three different curves correspond to different parametrizations of the symmetry energy.

\section{SUMMARY AND CONCLUSIONS}

In this work we studied neutron matter by combining pionless effective field theory at lowest order with non-perturbative lattice methods. To determine the neutron contact interaction we summed bubble chain diagrams contributing to neutron-neutron scattering at a given lattice spacing. The contact interaction was then adjusted to produce the pole in the amplitude indicated by Lüscher's finite volume formula for the physical ${ }^{1} S_{0}$ scattering length. Having determined the interaction coefficient for various lattice spacings, we then simulated neutron matter on the lattice using hybrid Monte Carlo at temperatures 4 and 8 $\mathrm{MeV}$ and densities below one-fifth normal nuclear matter density.

We find that our results at different lattice spacings agree with one another. This suggests that the continuum limit exists and that our effective theory was properly renormalized or, more conservatively, that any cutoff dependence is numerically small. For the range of parameters studied in this work we observe no instabilities towards phase separation, or towards lattice artifacts such as a completely filled lattice. While not unexpected, this is not a trivial result since the non-perturbative simulation includes all possible diagrams.

The energy per particle at temperatures $T=4 \mathrm{MeV}$ and $T=8 \mathrm{MeV}$ shows a steep downward slope at very small density, which is a sign of the strong attractive interaction 


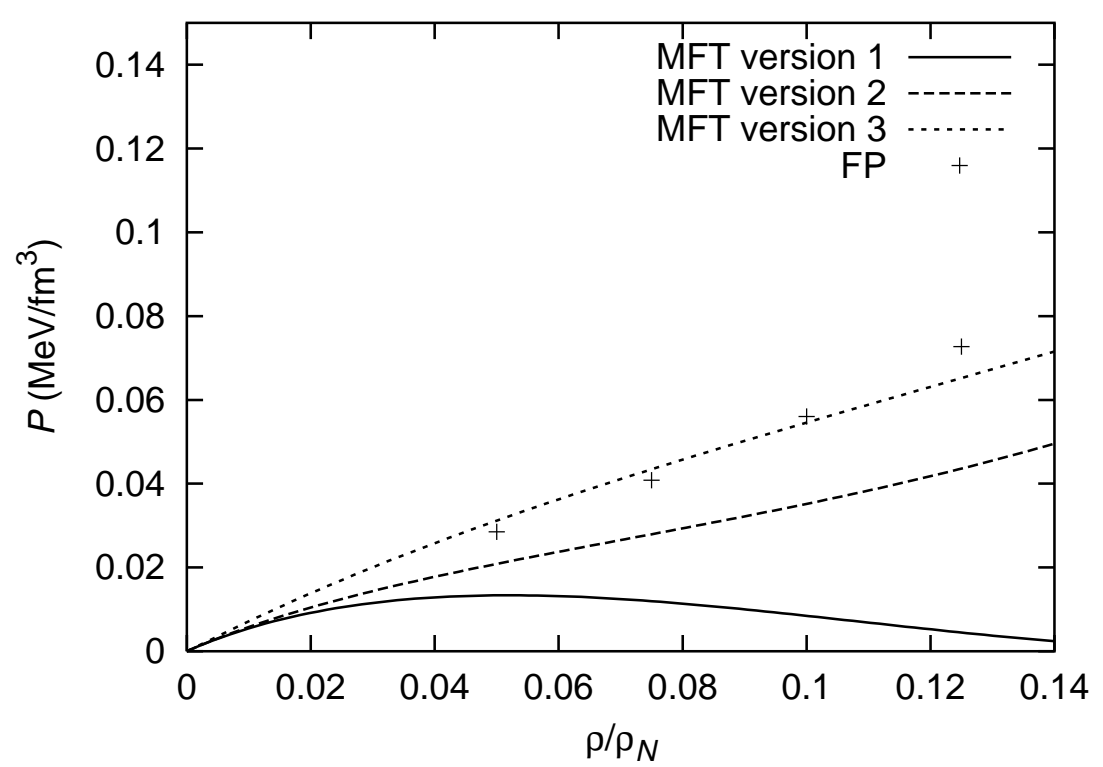

FIG. 13: Pressure versus density at $T=4 \mathrm{MeV}$ for the phenomenological equation of state discussed in [23]. The three different curves correspond to different parametrizations of the symmetry energy.

between neutrons. At intermediate densities $\rho \sim 0.1 \rho_{N}$ the energy per particle levels off. This behavior is reproduced quantitatively by our bubble chain calculations for $T>T_{F}$. In the future we wish to push our simulations to lower temperatures and determine the universal parameters $\xi$ and $\zeta$ for the energy per particle and gap as defined in (11). Simulations in this regime will require a source term for the di-neutron field. We have not seen unambiguous signs of superfluidity in our simulations. We did observe, however, a significant drop in HMC acceptance rate for the simulation at the lowest temperature and highest density studied in this work. This may well be an indication for the onset of superfluidity.

Our results for the pressure of pure neutron matter agree remarkably well with the variational calculation of Friedman and Pandharipande [9]. In the future it will be interesting to study whether the agreement persists if higher order terms in the effective Lagrangian or explicit pions are introduced. We have also performed simulations with explicit pions [17], but these data were taken at larger density and temperature. It will also be interesting to study systems with a finite proton fraction. This is easiest in the limit of exact Wigner symmetry, as the leading order Euclidean action is positive in that case [25].

It will also be interesting to investigate the phase structure of the leading order effective theory in more detail. In order to have a positive Euclidean action the coefficient $C$ of the 
four-fermion interaction has to be negative. This corresponds to either a negative scattering length or scattering length that is large and positive [18]. This implies that the effective theory studied in this work can be used to investigate the BCS-BEC crossover in a dilute Fermi gas.

\section{Acknowledgments}

The authors thank Simon Hands and Matthew Wingate for discussions on the hybrid Monte Carlo algorithm. This work was supported in part by DOE grants DE-FG88ER40388 and DE-FG02-04ER41335.

[1] H. Heiselberg and V. Pandharipande, Ann. Rev. Nucl. Part. Sci. 50, 481 (2000), astro$\mathrm{ph} / 0003276$.

[2] J. M. Lattimer and M. Prakash, Astrophys. J. 550, 426 (2001), astro-ph/0002232.

[3] D. J. Dean and M. Hjorth-Jensen, Rev. Mod. Phys. 75, 607 (2003), nucl-th/0210033.

[4] K. M. O'Hara, S. L. Hemmer, M. E. Gehm, S. R. Granade, and J. E. Thomas, Science 298, 2179 (2002).

[5] S. Gupta et al., Science 300, 1723 (2003).

[6] C. A. Regal and D. S. Jin, Phys. Rev. Lett. 90, 230404 (2003).

[7] T. Bourdel et al., Phys. Rev. Lett. 91, 020402 (2003).

[8] M. E. Gehm, S. L. Hemmer, S. R. Granade, K. M. O’Hara, and J. E. Thomas, Phys. Rev. A68, 011401(R) (2003).

[9] B. Friedman and V. R. Pandharipande, Nucl. Phys. A361, 502 (1981).

[10] J. Carlson, J. Morales, J., V. R. Pandharipande, and D. G. Ravenhall, Phys. Rev. C68, 025802 (2003), nucl-th/0302041.

[11] S. Weinberg, Phys. Lett. B251, 288 (1990).

[12] E. Epelbaum, W. Glockle, A. Kruger, and U.-G. Meissner, Nucl. Phys. A645, 413 (1999), nucl-th/9809084.

[13] S. R. Beane, P. F. Bedaque, W. C. Haxton, D. R. Phillips, and M. J. Savage, (2000), nuclth/0008064. 
[14] P. F. Bedaque and U. van Kolck, Ann. Rev. Nucl. Part. Sci. 52, 339 (2002), nucl-th/0203055.

[15] N. Kaiser, S. Fritsch, and W. Weise, Nucl. Phys. A697, 255 (2002), nucl-th/0105057.

[16] H. M. Müller, S. E. Koonin, R. Seki, and U. van Kolck, Phys. Rev. C61, 044320 (2000), nucl-th/9910038.

[17] D. Lee, B. Borasoy, and T. Schaefer, Phys. Rev. C70, 014007 (2004), nucl-th/0402072.

[18] J.-W. Chen and D. B. Kaplan, Phys. Rev. Lett. 92, 257002 (2004), hep-lat/0308016.

[19] M. Wingate, (2004), hep-lat/0409060.

[20] M. Lüscher, Commun. Math. Phys. 105, 153 (1986).

[21] S. R. Beane, P. F. Bedaque, A. Parreno, and M. J. Savage, (2003), hep-lat/0312004.

[22] S. Duane, A. D. Kennedy, B. J. Pendleton, and D. Roweth, Phys. Lett. B195, 216 (1987).

[23] B.-A. Li and C. M. Ko, Nucl. Phys. A618, 498 (1997), nucl-th/9701049.

[24] B.-A. Li, C. M. Ko, and W. Bauer, Int. J. Mod. Phys. E7, 147 (1998), nucl-th/9707014.

[25] J.-W. Chen, D. Lee, and T. Schaefer, (2004), nucl-th/0408043. 